\title{
Über Naphtindolinbasen
}

von

Josef Zangerle.

Aus dem chemischen Laboratorium der $k . k$. Universität in Innsbruck.

(Vorgelegt in der Sitzung am 3. Februar 1910.)

Die von K. Brunner ${ }^{1}$ aufgefundene Reaktion, wonach Phenylhydrazone von Aldehyden und Ketonen, welche die Isopropylgruppe enthalten, bei der Einwirkung alkoholischer Lösungen von Chlorzink oder von Zinnchlorür und Chlorwasserstoff oder von Jodwasserstoffsäure meist schon bei Zimmertemperatur unter Abspaltung von Ammoniak in Basen der Indolreihe, in Indoline übergehen, legte die Untersuchung nahe, ob diese Reaktion auch auf die Naphtylhydrazone dieser Art ausgedehnt werden könne.

Analog der von $\mathrm{K}$. Brunner beobachteten Bildung der E. Fischer'schen Base aus dem Methylphenylhydrazon des Isopropylmethylketons war für das $\alpha$-Naphtylhydrazon desselben Isopropylmethylketons die Bildung einer Indolinbase nach folgendem Prozeß zu erwarten:

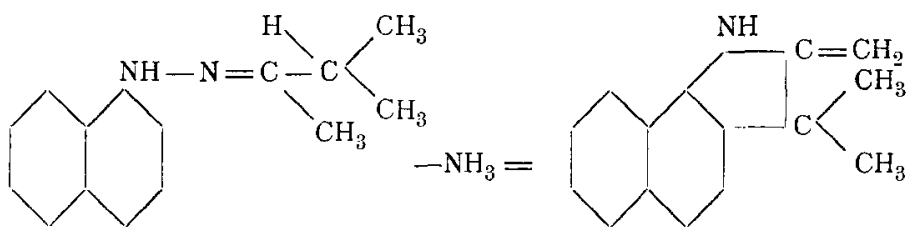

Das als Ausgangsprodukt benützte $\alpha$-Naphtylhydrazin stellte ich mir aus dem käuflichen salzsauren $\alpha$-Naphtylhydrazin nach den Angaben D. Li e be r's ${ }^{2}$ her. So dargestelltes $\alpha$-Naphtyl-

1 Monatshefte für Chemie, 16, 849 (1895); 17, 253 (1896); 21, 156 (1900).

2 Ebenda, 29, 422 (1008). 
hydrazin wurde in wenig Benzol gelöst, nötigenfalls filtriert, und mit etwas mehr als der äquimolekularen Menge Methylisopropylketon zusammengebracht. Nach längerem Stehen bei Zimmertemperatur und einstündigem Erwärmen am Wasserbad war die Reaktion beendet. Die gewonnene Lösung des Hydrazons wurde vom gebildeten Wasser abgegossen und mit wasserfreiem kohlensauren Kali getrocknet. Hierauf entfernte ich die größte Menge des Lösungsmittels und des überschüssigen Ketons durch Destillation am Wasserbad, anfangs bei gewöhnlichem, endlich unter vermindertem Druck, wobei ich einen ganz schwachen Luftstrom, der durch alkalische Pyrogallussäure und Schwefelsäure von Sauerstoff und Feuchtigkeit befreit war, hindurchleitete. Das zurückbleibende Hydrazon stellte ein tiefrot gefärbtes, zähflüssiges Öl dar, das nicht zur Krystallisation gebracht werden konnte.

Nach einem erfolglosen Versuch, das Hydrazon durch Erwärmen mit einer alkoholischen Chlorzinklösung in die Indolinbase überzuführen, ließ mich folgendes Verfahren zu derselben gelangen.

\section{Pr-3-3-Dimethyl-2-Methylen- $\alpha-$ Naphtindolin.}

Zur Darstellung dieser Base wurde zu einem Gewichtsteil Hydrazon eine vorher abgekühlte Lösung von einem Teil geschmolzenen Zinnchlorürs in gleichen Teilen 96 prozentigem Alkohol und Salzsäure vom spezifischen Gewicht 1.19 allmählich zugegossen. Die Mischung wurde im geschlossenen Kolben zunächst bei Zimmertemperatur mehrere Tage stehen gelassen, dann eine Stunde hindurch auf dem Wasserbad am Rückflußkühler erwärmt. Nach dem Abkühlen schieden sich reichlich Krystalle ab, die sich auf Zugabe von viel Äther noch vermehrten.

Die Krystalle bestehen aus einem Zinnchlorürdoppelsalze der Base und von Ammoniak. Sie wurden auf der Saugplatte gesammelt, mit Äther gewaschen und dann mit Wasser und Lauge zersetzt. Die Mischung schüttelte ich mehrmals mit Äther aus. Die ätherische Lösung behandelte ich mit verdünnter Schwefelsäure, um die Base von dem gleichzeitig 
entstandenen und etwa hier noch vorhandenen Indol ${ }^{1} \mathrm{zu}$ trennen. Die saure Lösung wurde mit Natronlauge zersetzt, die Base mit Äther aufgenommen und die ätherische Lösung getrocknet. Nach dem Verdunsten des Lösungsmittels blieb die Base krysallisiert zurück.

Die Base wurde zur Reinigung öfters aus Petroläther umkrystallisiert und zeigte nach dem Trocknen einen Schmelzpunkt von 70 bis $71^{\circ}$.

Die Analyse der gereinigten Base ergab folgende Werte:

I. $0.3007 \mathrm{~g}$ Substanz gaben $0.9467 \mathrm{~g}$ Kohlendioxyd und $0.198 \mathrm{~g}$ Wasser.

II. $0.3912 \mathrm{~g}$ Substanz gaben bei $15^{\circ}$ und $704 \mathrm{~mm}$ Druck $25.5 \mathrm{~cm}^{3}$ feuchten Stickstoff.

In 100 Teilen:

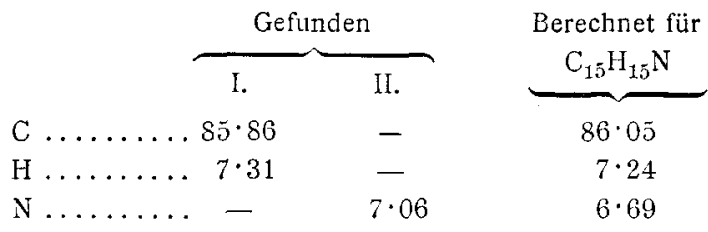

Die Base ist leicht löslich in Äther, in Alkohol und in Säuren.

\section{Platinchloriddoppelsalz.}

Aus salzsaurer, kalt bereiteter Lösung der Base fiel auf Zusatz von Platinchlorid sofort ein Niederschlag, der nach dem Waschen und Trocknen bei der quantitativen Bestimmung Werte gab, die mit den vermuteten Zahlen folgende Übereinstimmung zeigten:

$0.3471 g$ Substanz gaben $0.0811 g$ Platin.

In 100 Teilen:

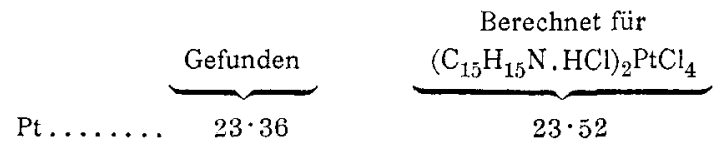

1 Die Menge des Indols, das nicht näher untersucht wurde und dessen Bildung immer bemerkbar war, übertraf namentlich bei sofortigem Erwärmen der Mischung die Menge der erhaltenen Base. 


\section{Pikrat.}

Beim Vermischen einer ätherischen Lösung der Base mit in Äther gelöster Pikrinsäure fiel ein rotgelber, schmutziger Niederschlag. Beim Versuch, diesen aus heißem Alkohol umzukrystallisieren, fiel immer wieder ein unreiner Körper, erst nach öfterem Umkrystallisieren aus heißem Benzol erhielt ich reine, dunkelgelbe Krystalle, die nach dem Trocknen einen konstanten Schmelzpunkt zeigten, der bei 149 bis $150^{\circ}$ lag.

Die Elementaranalyse ergab:

I. $0.2361 \mathrm{~g}$ Substanz gaben $0.5004 \mathrm{~g}$ Kohlendioxyd und $0.0877 \mathrm{~g}$ Wasser.

II. $0.2608 \mathrm{~g}$ Substanz gaben bei $19^{\circ}$ und $719 \mathrm{~mm}$ Druck $31.06 \mathrm{~cm}^{3}$ feuchten Stickstoff.

In 100 Teilen:

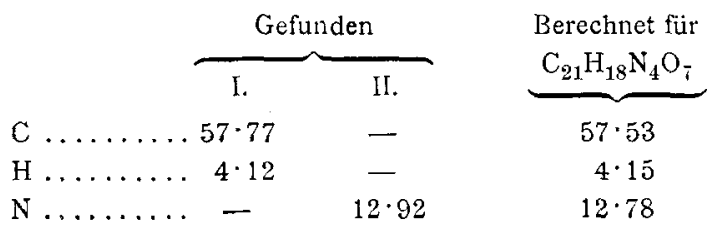

Wird die Base in verdünnter Salzsäure gelöst und mit Ferrocyankalium versetzt, so scheidet sich ein graues, an der Luft oberflächlich blau werdendes Salz ab.

Mit Zinnchlorid und konzentrierter Salzsäure scheidet sich ein rötlichgelbes Zinnsalz ab.

Mit Quecksilberchlorid entsteht ein schön.krystallisierendes Quecksilbersalz, welches sich in kleinen weißen Nadeln abscheidet.

\section{Methylierung der Base.}

Da eine sekundäre Base vorliegt, war vorauszusehen, daß. sich durch Einführen einer weiteren Methylgruppe eine neue Base herstellen lasse. Um dies zu erreichen, wurden $2 \cdot 3 \mathrm{~g}$ Base in $2.76 \mathrm{~cm}^{3}$ Alkohol gelöst und mit $1.15 \mathrm{~cm}^{3}$ Jodmethyl zusammengebracht. Nach längerem Erwärmen am Rückflußkühler schied sich ein rötlich gefärbtes Jodid $a b$, dessen Menge sich beim Abkühlen wesentlich vermehrte. Das gebildete Jodid wurde zur Entfernung der unverändert gebliebenen sekundären 
und der geringen Menge neugebildeter tertiärer Base mit Äther gewaschen und aus Alkohol unter Zugabe einiger Tropfen schwefeliger Säure einigemale umkrystallisiert, ohne einen konstanten Schmelzpunkt erreichen zu können. Daher löste ich das Jodid in Alkohol und fällte es wieder mit relativ viel Äther aus. ${ }^{1}$ Jetzt erst zeigten die Krystalle nach oftmaligem Umkrystallisieren aus Alkohol einen konstanten Schmelzpunkt, der bei $229^{\circ}$ liegt.

Die Elementaranalyse dieses Salzes gab mit den erwarteten Zahlen folgende Übereinstimmung:

I. $0.2887 \mathrm{~g}$ Substanz gaben $0.576 \mathrm{~g}$ Kohlendioxyd und $0.1351 \mathrm{~g}$ Wasser.

II. $0.3254 \mathrm{~g}$ Substanz gaben bei $15^{\circ}$ und $709 \mathrm{~mm}$ Druck $12.61 \mathrm{~cm}^{3}$ feuchten Stickstoff.

III. $0 \cdot 2035 g$ Substanz gaben $0 \cdot 1335 g$ Jodsilber.

In 100 Teilen:

$\begin{array}{ccccc}\text { I. } & \text { II. } & \text { III. } & \underbrace{\text { Gefunden }} & \begin{array}{c}\text { Berechnet für } \\ \mathrm{C}_{16} \mathrm{H}_{18} \mathrm{NJ}\end{array} \\ \mathrm{H} \ldots \ldots \ldots & 54 \cdot 38 & - & - & 54 \cdot 7 \\ \mathrm{~N} \ldots \ldots \ldots & 5 \cdot 19 & - & - & 5 \cdot 12 \\ \mathrm{~J} \ldots \ldots \ldots & - & 4 \cdot 22 & - & 3 \cdot 98 \\ & - & 35 \cdot 95 & 36 \cdot 13\end{array}$

\section{Pr-1-n-Methyl-3-3-Dimethyl-2-Methylen- $\alpha$-Naphtindolin.}

Um die Base zu gewinnen, zersetzte ich ihr Jodid mit einem Überschuß von Kalilauge und nahm sie in Äther auf. Die mit entwässerter Pottasche getrocknete Lösung hinterlie 3 beim Verdunsten die Base als dunkelblau gefärbtes Öl. Dieses konnte nicht zur Krystallisation gebracht werden.

Die Salze dieser Base krystallisieren noch schöner als die der unmethylierten.

So fällt das Pikrat nach der Vereinigung der ätherischen Lösungen der Pikrinsäure und der Base sofort in schönen, hellgelben Krystallen. Diese zeigen einen Schmelzpunkt von $177^{\circ}$.

Die salzsaure Lösung der Base gibt mit konzentrierter Eisenchloridlösung nach Zugabe von konzentrierter Salzsäure

1 Vergl. A, Konschegg, Monatshefte für Chemie, 27, 250 (1906). 
ein krystallisiertes Eisensalz und mit Platinchlorid ein Platindoppelsalz.

Die Base zeigt wie die Fischer'sche Base das charakteristische Verhalten, sich an der Luft zu färben und kann für sie in Übereinstimmung mit obiger Nomenklatur folgende Strukturformel aufgestellt werden:

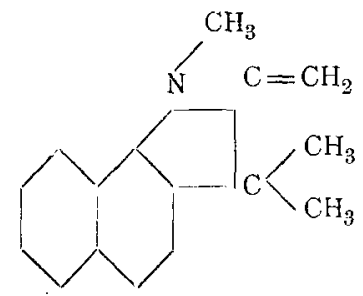

\section{Darstellung des Pr-3-3-Dimethyl-2-Methylen- $\beta$-Naphtindolins.}

Auch zur Darstellung dieses zum vorhin beschriebenen stellungsisomeren Indolins ging ich bei den ersten Versuchen von dem salzsauren Salze des $\beta$-Naphtylhydrazins aus, später verwendete ich aber das freie $\beta$-Naphtylhydrazin, wie es jetzt Kahlbaum in zugeschmolzenen Glaskugeln in den Handel bringt.

Das freie Hydrazin wurde wieder in möglichst wenig Benzol in der Wärme gelöst und mit der berechneten Menge Isopropylmethylketon erwärmt. Die Bildung des Hydrazons ging in ganz analoger Weise vor sich wie bei der $\alpha$-Verbindung. Die Darstellung des Indolins aus dem Hydrazon durch Behandeln mit Zinnchlorür gelang aber trotz vielfacher Versuche nicht oder wenigstens sehr schlecht. Beim Zusammenbringen des Hydrazons mit der alkoholischen Zinnchlorürlösung und Salzsäure trat selbst in der Kälte eine heftige Reaktion ein, wonach die ganze Masse sofort zu einem festen. Klumpen erstarrte. Der Geruch nach Isopropylmethylketon, der hierbei auftrat, ließ mich auf eine Zersetzung schließen und diese Vermutung wurde bei der Untersuchung des Reaktionsproduktes bestätigt.

Ich wandte daher ein anderes Verfahren an, das, wie mir Prof. K. Brunner mitteilte, nach seinen Versuchen auch die Bildung von Indolinbasen aus Hydrazonen zuließ und in diesem Falle, wo Mineralsäuren zu heftig reagierten, Erfolg versprach. 
Dasselbe besteht in der Anwendung einer alkoholischen Lösung von wasserfreier Oxalsäure. Zu $1 g$ Hydrazon wurden $2 g$ entwässerte Oxalsäure, in $4 \mathrm{~g}$ Alkohol gelöst, langsam zugegossen. Wird die alkoholische Oxalsäurelösung rasch zugegeben, so fällt sofort ein dichter Niederschlag und beim Kühlen erstarrt die ganze Masse, sie wird aber bei längerem Stehen bei gewöhnlicher Temperatur allmählich wieder flüssig. Ich ließ die Oxalsäure 2 Tage einwirken, verdünnte dann das Reaktionsprodukt mit Wasser und machte mit Kalilauge alkalisch. Dann schüttelte ich mit Äther gut durch und entfernte das gebildete Indol auf früher angegebene Weise. Nach dem Trocknen und Verdunsten des Lösungsmittels blieb die Base krystallisiert zurück. Die Ausbeute an unreiner Base beträgt ungefähr 50\% der berechneten. Das Umkrystallisieren der Base aus verdünntem Alkohol gelingt sehr schwer, da sich zuerst immer ein unreines Produkt abscheidet. Bei der Destillation der Base mit Wasserdampf erhielt ich nur geringe Ausbeuten. Besser gelingt die Reinigung durch Überführen der Base in ihr Jodid.

\section{Jodhydrat der Base.}

Zu diesem $Z$ wecke löste ich die Base in verdünnter Salzsäure, filtrierte und versetzte mit einer Jodkaliumlösung; nach längerem Stehen schieden sich reichlich nadelförmige, fast farblose Krystalle aus. Das Jodid wird durch Umkrystallisieren aus heißem Wasser gereinigt und zeigt dann einen Schmelzpunkt, der bei 224 bis $225^{\circ}$ liegt. Werte:

Die Elementaranalyse dieses Körpers ergab folgende

I. $0.3279 \mathrm{~g}$ Substanz gaben $0.6416 \mathrm{~g}$ Kohlensäure und $0 \cdot 1400 \mathrm{~g}$ Wasser.

II. $1.0 \cdot 2780 \mathrm{~g}$ Substanz gaben $0 \cdot 1927 \mathrm{~g}$ Jodsilber.

2. $0.3729 \mathrm{~g}$ Substanz gaben $0.2591 \mathrm{~g}$ Jodsilber.

In 100 Teilen:

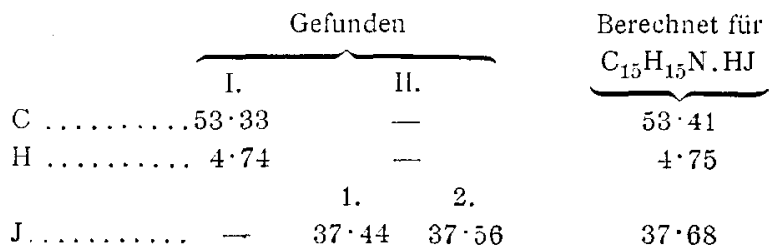




\section{Pr-3-3-Dimethyl-2-Methylen- $\beta-N a p h t i n d o l i n$.}

Die freie Base stellte ich aus dem Jodid dar, indem ich dieses mit Kalilauge zersetzte, die Base mit Äther ausschüttelte, die ätherische Lösung mit kohlensaurem Kali von Wasser befreite und das Lösungsmittel verdunstete. Zur vollständigen Reinigung wurde sie noch zweimal aus verdünntem Alkohol umkrystallisiert. Nach dem Waschen und Trocknen zeigt sie einen Schmelzpunkt von $115^{\circ}$ und ist identisch mit der von Fischer und Steche ${ }^{1}$ aus dem Pr-2-3-Dimethyl- $\beta$-Naphtindol durch Erhitzen mit Jodmethyl hergestellten Base.

Die Elementaranalyse ergab:

I. $0.2623 \mathrm{~g}$ Substanz gaben $0.8272 \mathrm{~g}$ Kohlensäure und $0.1751 \mathrm{~g}$ Wasser.

II. $0.4217 \mathrm{~g}$ Substanz gaben bei $17^{\circ}$ und $710 \mathrm{~mm}$ Druck $27 \mathrm{~cm}^{3}$ feuchten Stickstoff.

In 100 Teilen:

\begin{tabular}{|c|c|c|c|}
\hline & \multicolumn{2}{|c|}{ Gefunden } & \multirow{2}{*}{$\begin{array}{l}\begin{array}{c}\text { Berechnet für } \\
\mathrm{C}_{15} \mathrm{H}_{15} \mathrm{~N}\end{array} \\
\end{array}$} \\
\hline & I. & II. & \\
\hline $\mathrm{C}$ & $.86 \cdot 00$ & - & 86.05 \\
\hline $\mathrm{H}$ & $.7 \cdot 39$ & - & $7 \cdot 24$ \\
\hline $\mathrm{N}$ & - & $6 \cdot 92$ & 6.69 \\
\hline
\end{tabular}

Die Base ist in Alkohol und Äther leicht, in Wasser sehr schwer löslich.

Die Synthese der Base läßt für die Strukturformel die Wahl zwischen den beiden folgenden offen:

oder
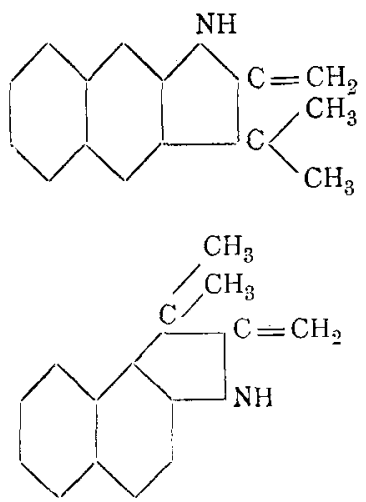

1 Bericht der Deutschen chem. Ges, 20,818 (1887); Annalen der Chemie, 242,364 . 


\section{Platinchloriddoppelsalz.}

Die Base wird in verdünnter Salzsäure gelöst und filtriert. Die Lösung gibt mit Platinchlorid sofort einen dichten Niederschlag, der nach dem Auswaschen und Trocknen ein hellgelbes Pulver darstelit.

Eine Platinbestimmung ergab:

$0 \cdot 4019 \mathrm{~g}$ Substanz gaben $0 \cdot 0937 \mathrm{~g}$ Platin.

In 100 Teilen:

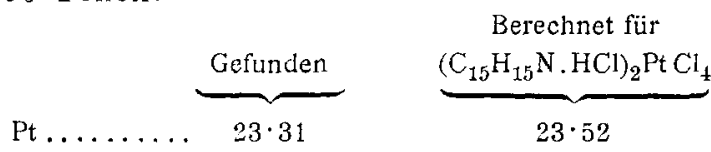

Diese Base gibt ebenfalls in ätherischer Lösung mit ätherischer Pikrinsäurelösung ein schön krystallisiertes Pikrat, dessen Schmelzpunkt bei 224 bis $226^{\circ}$ liegt.

Ein Acetylprodukt der Base bildet sich leicht beim halbstündigen Erwärmen mit Essigsüureanhydrid. Es fällt beim Verdünnen des Reaktionsproduktes mit Wasser in Form von öligen Tropfen, die auch in der Kälte nicht zur Krystallisation gebracht werden konnten. Nach dem Ausschütteln dieser Flüssigkeit mit Äther blieb aber nach dem Verdunsten des Lösungsmittels das Acetylprodukt krystallisiert zurück. Es wurde aus Petroleumäther umkrystallisiert. Sein Schmelzpunkt liegt bei $109^{\circ}$.

Desgleichen liefert die Base ein Benzoylprodukt. Wird nämlich die Base mit Kalilauge und Benzoylchlorid geschüttelt, so scheidet sich dieses in öligen Tropfen aus, die in der Kälte allmählich erstarren. Die Krystalle werden abgesaugt und aus verdünntem Alkohol umkrystailisiert. Die weißen plattenförmigen Krystalle zeigen einen Schmelzpunkt von $114^{\circ}$.

\section{Methylierung des Pr-3-3-Dimethyl-2-Methlyen- $\beta$-Napht- indolins.}

Beim Behandeln der $\beta$-Base mit Jodmethyl tritt schon in der Kälte eine Reaktion ein und nach einigen Stunden Stehens 
erstarrt die ganze Masse. Nach A. Pinner und A. Fran ${ }^{1}$ war bei der Methylierung kein einheitliches Produkt zu erwarten, da sich neben jodwasserstoffsaurem Salz der sekundären Base und der tertiären Base auch freie tertiäre Base bilden mußte. Daher wurde das Reaktionsgemisch vorerst mit Äther gewaschen. Das zurïckgebliebene Gemisch der beiden Jodide krystallisierte ich einigemale aus verdünntem Alkohol unter Zugabe einiger Tropfen schwefeliger Säure um. Das so erhaltene Produkt zeigte einen Schmelzpunkt von $224^{\circ}$. Der Schmelzpunkt ist mit dem des Jodids der unmethylierten Base identisch und eine quantitative Jodbestimmung legte die Gleichheit der beiden Körper untrüglich dar. $\mathrm{Zu}$ dem gleichen Resultat gelangte ich, wenn ich das Jodid in Alkohol löste und mit relativ viel Äther wieder fällte. Auch wenn ich das Jodid mit lauwarmem Wasser übergoß, krystallisierte mir aus der Lösung das gleiche Produkt.

Nun suchte ich die Base in dem ätherischen Auszug des Reaktionsgemisches. Nach dem Verdunsten des Äthers blieb ein krystallisierter Rückstand, der sich an der Luft grün und schließlich blau färbte und ein Gemisch von ursprünglicher und methylierter Base darstellte. Die methylierte Base durch Unkrystallisieren zu trennen, gelang nicht, sondern es resultierte nach oftmaligem Umkrystallisieren jedesmal ein Produkt, das mit der ursprünglichen Base identisch war, wie die gleichen Schmelzpunkte ergaben.

Eine Trennung der beiden Basen konnte ich erst erreichen, als ich von der Annahme ausging, daß sich die sekundäre Base von der etwa vorhandenen tertiären Base durch die Bitdung eines Nitrosoproduktes scheiden ließe.

Die Base wurde in verdünnter Salzsäure gelöst, filtriert und die Lösung mit einer Kaliumnitritlösung versetzt. Das sich sofort abscheidende Nitrosamin wurde in Äther aufgenommen und nach dem Verdunsten des mit kohlensaurem Kali getrockneten Lösungsmittels zweimal aus Benzol umkrystallisiert. Die erhaltenen rötlichgelben Krystalle zeigen einen Schmelzpunkt von 175 bis $176^{\circ}$.

Zur Methylierung der Base und Trennung des Reaktionsgemisches schlug ich nun folgenden Weg ein: $4 \mathrm{~g}$ der sekun-

1 Berichte der Deutschen chem. Ges., 38, 1539 (1905). 
dären Base brachte ich in $4 \mathrm{~cm}^{3}$ Jodmethyl durch längeres Schütteln in Lösung und ließ die Lösung 3 Tage stehen. Das erstarrte Reaktionsprodukt behandelte ich wieder mit Äther. Das gebildete Jodid zersetzte ich mit Kalilauge, schüttelte mit Äther gut durch und nach dem Verdunsten der beiden ätherischen Lösungen resultierte das Gemisch der beiden Basen. Dieses wurde in wenig verdünnter Salzsäure gelöst, die Lösung mit Wasser stark verdünnt und unter Kühlung langsam eine verdünnte Kaliumnitritlösung zugegeben. Das abgeschiedene Nitrosamin wurde in Äther aufgenommen und diese ätherische Lösung des Nitrosamins von der wässerigen Lösung des salzsauren Salzes der tertiären Base mit dem Scheidetrichter getrennt. Die wässerige Lösung schüttelte ich zweimal mit Äther gut durch und fällte daraus die tertiäre Base durch Übersättigen mit Kalilauge. Sie wurde in Äther aufgenommen und blieb nach dem Trocknen des Lösungsmittels mit entwässertem kohlensauren Kali und nach dem Verdunsten des Äthers krystallisiert zurück. Die Menge der erhaltenen Base ist eine sehr geringe. Sie wurde zweimal aus verdünntem Alkohol umkrystallisiert und zeigte dann einen Schmelzpunkt von 119 bis $120^{\circ}$.

Sie färbt sich an der Luft blau. Ich löste sie sofort in verdünnter Salzsäure und fällte aus der Lösung durch Zugabe einer Jodkaliumlösung ihr Jodid. Dieses krystallisierte aus verdünntem Alkohol in fast weißen Nadeln und zeigte dann einen Schmelzpunkt von $233^{\circ}$.

Eine quantitative Jodbestimmung dieses Produktes ergab Werte, die dessen Unterschied von dem Jodid der unmethylierten Base deutlich erkennen ließen: ${ }^{1}$

$0 \cdot 3300 g$ Substanz gaben $0.2207 g$ Jodsilber.

In 100 Teilen:

$$
\mathrm{J} \ldots \ldots \ldots \ldots \underbrace{\text { Gefunden }}_{36 \cdot 09} \quad \overbrace{\begin{array}{c}
\mathrm{C}_{16} \mathrm{H}_{18} \mathrm{NJ} \\
36 \cdot 13
\end{array}}^{\text {Berechnet für }} \begin{gathered}
\mathrm{C}_{15} \mathrm{H}_{16} \mathrm{NJ} \\
37 \cdot 68
\end{gathered}
$$

1 Eine Methylbestiinmung versprach keine sichere Entscheidung, da auch die unmethylierte Base, nach der Methode von J. Herzig und Hans Meyer behandelt, Methylabspaltung erkennen lieB. 
Die vorliegende Untersuchung ergab, daß sowohl $x$ - als $\beta$-Naphtylhydrazon des Isopropylmethylketons bei der Behandlung mit Säuren in alkoholischer Lösung zunächst sekundäre Methylenindolinbasen liefern, die dann mit Jodmethyl zum Teil in tertiäre Methylenindolinbasen übergehen. Letztere stellen die der E. Fischer'schen Base analogen Verbindungen der Naphtindolinreihe dar und zeigen wie die E. Fischer'sche Base die Eigenschaft, an der Luft eine intensive, jedoch nicht rote, sondern blauviolette Färbung anzunehmen. 